Mansoura University

Faculty of Education

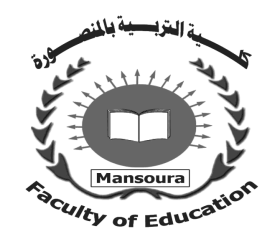

\title{
L'INTERFÉRENCE ET LA TRANSPOSITION DES TERMES JURIDIQUES DANS LE DROIT MUSULMAN
}

\author{
Recherche présentée par \\ Dr/ Kamal Ali Mahmoud Ahmed \\ Maître de conférences au département du français, Faculté de langues et de \\ traduction, Université d'Al Azhar, le Caire
}

Journal Of The Faculty Of Education-Mansoura University

No. 110 - April. 2020 


\title{
L'interférence et la transposition des termes juridiques dans le droit musulman
}

\author{
Dr/ Kamal Ali Mahmoud Ahmed \\ Maître de conférences au département du français, \\ Faculté de langues et de traduction, Université d'Al \\ Azhar, le Caire
}

\section{Introduction}

$\mathrm{Vu}$ les différences culturelles qui sont à l'origine de l'apparition des termes, surtout les termes techniques, n'importe quel terme ne pourrait remplir exactement sans notion que dans la langue qui l'a produit.

En ce qui concerne les termes juridiques il s'agit d'une sorte d'unanimité collective qui vient spontanément des exigences sociales

Il est connu que le droit moderne représenté d'abord dans le code de Napoléon, trouve ses origines dans le patrimoine juridique romain d'où le maintien des termes latins dans le code français jusqu'à présent ; citons à titre d'exemple stricto sensu, a priori, a fortiori, e facto, etc...

Pour le droit musulman il n'existe pas d'antécédent idiomatique car les arabes avant l'islam ne connaissaient pas les codes, leurs lois prenaient la forme des us et coutumes.

Lorsque les doctes ont procédé à légiférer les sentences islamiques, ils avaient inventaient leur lexique et leur glossaire par le biais de deux sources : la première est ce dynamisme que la langue arabe avait acquise en conséquences du progrès scientifique, la deuxième source était le Coran et la Sunna. La première question à poser : à quelle point est-il possible que des termes s'équivalent dans deux domaines voisins alors que le système lexical et le contexte culturel ne sont pas les mêmes.

Le droit renferme tous les domaines de la vie à partir du droit constitutionnel qui régit l'Etat, le régime politique, le gouvernement, le parlement, les élections etc...jusqu'aux lois relatives aux mœurs passant par le droit administratif, le droit le pénal, le droit civil, le droit du commerce. N'importe quel domaine de la vie doit être organisé par la loi. Le droit musulman renferme en plus les chapitres susmentionnés, les rapports entre les gens et leur Seigneur, ce qui donne un enrichissement davantage à notre étude

De ce qui précède, s'impose la problématique de notre recherche vis-àvis de cette divergence entre les deux sphères culturelles et les spécificités des deux contextes sociaux surtout devant une vérité disant que l'origine du droit musulman est quasiment religieuse alors que celui du droit positif est 
purement laïc, Est-il possible que la transposition des termes spécifiques de l'arabe vers le français se fasse avec pertinence ou bien laisse-t-elle beaucoup à dire?

Il est connu que le terme juridique est l'expression d'une notion à la fois absolue et exactement applicable à des cas déterminés de figures, Il y a des termes qui font confusion en raison de leurs sens voisins. Pourtant traduire n'importe quel terme par un autre à sens voisin pourrait causer une bonne partie de défaillance sémantique et par conséquent un chaos juridique.

Si on y ajoute l'écart socioculturel entre les deux langues : cible et source apparaitra l'importance de cette étude par exemple le terme (imam) dans le chapitre des actes culturels signifie celui qui dirige la prière, dans le droit constitutionnel c'est le gouverneur ou le calife. Pour bien traduire ces termes il faut connaitre exactement et précisément la notion du terme dans son pays

Une question se pose : pourquoi traduire le droit musulman en français ? Avant de répondre à cette question il faut signaler que les orientalistes eux-mêmes ont effectué une traduction de la plupart des livres musulmans dans le cadre de connaitre l'autre avec qui les rapports sont indispensables soient-t-ils pacifique ou conflictuels. On y ajoute l'existence des millions des musulmans en France sans oublier les rapports entre la France et les pays musulmans. Si on renonce à la traduction du droit musulman en français, on risque de se trouver devant des traductions défectueuses effectués soit par des amateurs ou par des malhonnêtes ou tendancieux alors à ce moment-là la traduction devient une exigence scientifique et une obligation religieuse pour bien présenter notre patrimoine islamique au non-musulmans.

Notre travail au cours de cette recherche consistera d'abord à la prise des échantillons des termes du droit musulman publique qui renferme les sentences et prescriptions relatives au califat et statut gouvernemental. Nous allons faire la transcription de ses termes en caractère latin puis leur donner la signification exacte dans le droit musulman. Ensuite nous allons procéder à examiner les équivalents français donnés à ces termes pour donner un jugement de valeur sur la pertinence de la traduction principalement basée sur la bonne compréhension de la part du traducteur du patrimoine juridique musulman.

Finalement, on entend faire une comparaison entre les termes du droit musulman et ceux du droit positif et aussi la différenciation des notions juridiques relevées du droit musulman 
Pour mettre en exergue les particularités conceptuelles du droit musulman nous allons aborder les problèmes des termes juridiques du point de vue à la fois linguistique et socioculturel à travers une application sur le livre le califat d'Assanhoury

Notre étude est destinée également à répondre aux questions suivantes :

$>$ quels sont les problèmes théoriques et pratiques de la traduction spécifique?

$>$ Quels sont les inconvénients affrontés par le traducteur en effectuant une traduction juridique?

Quels sont les rapports entre le terme et le contexte culturel ?

$>$ Assanhoury a- il réussit à bien établir l'équivalence entre le terme du droit musulman et celui du droit positif?

$>$ Les remarques qu'il a émises sur la traduction d'Orstrorog sont t-elles pertinentes?

$>$ Quelles sont les exigences nécessaires pour bien traduire les termes du droit musulman?

$>$ Est-ce que les traducteurs occidentaux ont-t- ils réussi vraiment à faire une traduction pertinente du vouloir dire des doctes musulmans?

$>$ Jusqu'à quel point ont-ils eu recours à faire des traductions approchantes?

Premiere Partie

La traduction spécifique (Approche juridique)

Premier Chapitre

Les particularités de la traduction juridique

La traduction spécifique est cette traduction qui porte sur des domaines scientifiques dans des disciplines exactes : médicine, sciences humaines, droit, sciences religieuses, sociologie etc....

Cette sorte de traduction est radicalement différente de la traduction des textes ordinaires (de presse, des dissertations, langage parlés, etc...

$\mathrm{Si}$ la traduction en général exige une maitrise et une bonne connaissance de deux langues : source et cible ; la traduction spécifique nécessite impérativement une sorte d'érudition dans le domaine auquel révèle l'ouvrage à traduire. Par conséquent il est préférable qu'un spécialiste dans la discipline procède à effectuer la traduction des ouvrages appartenant à son domaine; c'est parce qu'il maitrise bien les particularités et les spécificités de la discipline beaucoup mieux que le simple traducteur.

Etant donné que cela n'est pas toujours disponible, faute d'un nombre suffisant de traducteurs spécialistes et également parce qu'il y a plusieurs savants qui, soit ne maitrisent pas une langue étrangère, soit ils ne sont pas 
doués de traduire. Ainsi, incombe sur les traducteurs une mission lourde ; ils sont invités à transmettre toute une connaissance colossale de la langue étrangère et de leur langue maternelle.

Actuellement la traduction n'est pas un travail ni de plaisir ni de luxe, elle est devenue plutôt une industrie, voire le seul véhicule de la culture et le moyen primordial de la civilisation.

(La traduction spécifique et surtout juridique répond à des nécessités pratiques impérieuses qu'il est possible d'envisager successivement, et sans la lumière de la traduction juridique on ne trouvera beaucoup d'observations) ${ }^{(1)}$

L'importance de la traduction spécifique s'accroit surtout pour trois raisons : primo, la plupart des étudiants des disciplines spécifiques surtout dans les pays arabes ne maitrisent pas la langue étrangère et par conséquent ils sont incapables de se tenir au courant de ce qui est à jour dans leur discipline. Secundo, la traduction contribue à une acculturation et les échanges des connaissances entre les diverses contrées. Tirso, la traduction des sciences humaines fait un rapprochement entre les divers peuples surtout lorsqu'il s'agit de quelque chose de patrimonial.

Pour la traduction juridique elle se tient parmi les genres les plus importants de la traduction spécifique dans les quatre coins du monde, pourquoi ? 1- les lois et les législations de n'importe quelle société nous montre plusieurs choses dont : sa culture, sa manière de raisonne, ses traditions, ses propriétés, son régime, etc. 2- le monde est devenu un village planétaire d'où l'effacement des barrières, les medias modernes, les moyens de communications, très immédiats et par conséquent ce qui intéresse un peuple s'inscrit forcement dans les préoccupations des autres peuples : comment connaitre un peuple sans prendre en considération son système juridique. 3- Le mouvement du commerce dans le monde entier est devenu transfrontalier et transcontinentaux d'où l'importance de connaitre parfaitement les diverses législations économiques, financières, juridiques, monétaires et commerciales de tous les pays surtout vu le GATT, les organisations commerciales, régionales et mondiales et la nécessite de s'adapter aux exigences de la réalité vécu. 4- Lorsqu'il s'agit d'une traduction juridique qui porte sur des ouvrages d'ordre religieux, le besoin devient plus accru surtout pour se mettre en bonne entente avec les cultures des autres peuples. $\mathrm{Au}$ cours $\mathrm{du}$ premier chapitre nous entendons traiter les

(1) Brèves notes sur les problèmes de la traduction juridique - Revue internationale de droit comparé Année 1986 Volume 38 Numéro 2 pp. 349 
particularités de la traduction juridique et par la suite les difficultés effectuées une telle traduction et comment les surmontes $?^{(1)}$

Le droit est l'outil, l'instrument et le procédé responsables d'organiser la vie d'une société, les rapports entre les individus et les citoyens de l'Etat. Le droit musulman, à l'instar de tous les systèmes juridiques est le miroir d'une société dynamique et évolutive. Il est passé par des diverses étapes comme suit :

La première étape : eut lieu à l'époque du prophète où les termes ont été inspirés par le Coran et la sunna citant à titre d'exemple, il y avait les termes comme : salat, zakat, seyam et hajj et d'autres termes cultuels lorsque un non-musulman procède à traduire ces termes en langue autre que l'arabe, le français par exemple, il se trouvera devant une difficulté d'interprétation.

Dans la Bible (Ancien et Nouveau testament), Il y a une salat dans le sens de la prière, une zakat dans le sens de l'aumône et la charité et un seyam dans le sens du carême et le jeune mais est ce que les termes musulmans ont les mêmes significations des termes bibliques ? C'est-à-dire la signification des énoncés biblique remplie-t-elle le vouloir dire des termes islamiques ? la réponse est certainement négative et nous allons voir tout de suite la raison selon une petite application relative a quelques termes islamiques traduit en littéralement comme les termes biblique et enfin nous nous trouvons devant une mauvaise qui sera sans aucun doute la cause d'une fausse compréhension ; commençant par (la salat) en islam est carrément différente de la notion de la prière dans la bible.

(le seyam) en islam consiste à s'abstenir strictement de toute nourriture, toute boisson et tout rapport charnels de l'aube jusqu'au couche du soleil et lors de la rupture du seyam (iftar) tout redevient permis tout au long de la nuit et ainsi de suite pour un mois entier $)^{(2)}$

Alors que la carême ou le jeune signifie de s'abstenir de certains nourriture jour et nuit pour plus de deux mois, on pourrait manger et boire de ce qui n'est pas interdit alors le seyam n'est pas la carême .

(Pour (la zakat) elle est d'abord prescrite ensuite conditionnée et finalement la proportion est bien déterminée et les bénéficiaires également)

Alors que l'aumône ou la charité est d'abord facultative puis n'est pas conditionnée et finalement c'est l'église qui la collecte et par conséquent la zakat n'est pas l'aumône.

(1) Ibid., p. 350

(2) Al-Sayed Sabek Fekeh El-Sunna - premier chapitre ,p. 235 
Pour les transactions, la vente, l'achat, la location ...etc. Elles sont presque les mêmes. Même l'intérêt usuraire (Reba) est prohibé dans la Bible que dans le Coran et par conséquent la traduction de ce qu'on appelle la jurisprudence de transaction (Fiqh el màamallât) vers les langues étrangères est tout à fait faisable, certes il y a des difficultés, mais on pourrait facilement les surmontes, il faut signaler à ce propos que

(Le droit musulman ne s'est cristallisé que sous le mouvement scientifique après la mort du prophète et l'ouverture des musulmans sur les autres communistes et cultures raison pour laquelle les termes de transaction devraient être accessibles et compréhensible aussi bien pour les musulmans que pour les non-musulmans.) ${ }^{(1)}$

Passant aux exigences sociales; nous pourrons dire que le langage du droit musulman lors de son élaboration se caractérisaient par certaines particularités, dont en tête :

1. un langage très soutenu, standard, et revêtu d'une éloquence exemplaire, à comprendre a peine pour l'arabe ou l'arabophone ordinaire.

2. il s'agit d'un langage très précis et très spécifique, La mal compréhension d'un seul terme ou même une préposition est susceptible de rendre la traduction ambiguë 3-Il existe des termes qui sont purement arabes et qui n'ont pas d'équivalent dans la langue française.

Toutes les caractéristiques susmentionnés rendent difficile la traduction du droit musulman vers le français. Nous pouvons donner un exemple qui met en évidence la difficulté de la mission du traducteur de cette discipline :

$$
\text { (سلامة الاعضاء من نقص يمنع عن استيفاء من حركة و سرعة النهوض) (lexpts ) }
$$

(La santé des membres du corps tellement qu'ils soient exempts de tous défauts pouvant empêcher la liberté et la promptitude des mouvements) ${ }^{(2)}$

Cette longue phrase de la part du traducteur (Ostrorog) est la traduction d'une phrase courte et concise d'Al-Mawardy. il a bien employé le terme (membre). L'emploi du terme (liberté) est ici confus, Pourquoi ? Parce que dans le droit musulman on fait toujours la distinction entre la liberté et l'esclavage, L'esclavage a un statut très diffère de celui de l'homme libre. Le traducteur devait dire la capacité de bien bouger et agir au lieu de

(1) Al-Sayed Sabek Fekeh El-Sunna) - Deuxième chapitre, p. 390

(2) Al-Mawardi : Al-Ahkam Al-Sultaneyah- p.4 (traduction d'Ostrorog p.102) 
la liberté. /e mot (لنهوض) veut dire dans ce contexte ( se lever ) sans peine et surtout accomplir sa mission alors que le traducteur a mal interprété le vouloir dire d'Al-Mawardy parce qu'il a compris que le mot (النهوض) est dans le sens de ( se mouvoir ).

Le droit français emploie les deux termes jurisprudence et doctrine (Fiqh) et (mazhab) et les deux termes se trouvent en arabe. Au premier abord, nous croyons que le mot jurisprudence veut dire la production juridique islamique et le mot doctrine veut dire l'école juridique comme l'école Hanafite, Shaféite ; etc....

L'examen soigneux et attentif de ces deux termes nous révèlent ce qui suit : le mot (Fiqh) en arabe signifie l'ensemble de la production juridique élaboré par les doctes musulmans, il est d'un caractère académique et scientifique et non pas forcement juridictionnel alors que la jurisprudence dans le droit moderne est le bilan de la pratique juridictionnel des cours suprême : on dit par exemple la jurisprudence de la cour de cassation. Quant à la doctrine ; il s'agit des théories et conclusions juridiques effectues par les professeurs de droit mais en islam (Al-Mazhab) représente les grandes lignes et tendances d'une école juridique et non pas d'une seule personne, donc traduire (le Fiqh) par jurisprudence et (le mazhab) par doctrine n'est pas exacte. On y ajoute que le mot doctrine peut remplir le sens (Akida)

\section{Deuxieme Chapitre}

\section{(Surmonter les problèmes de la traduction spécifique)}

La pluralité des problèmes de la traduction juridique dans le droit musulman nous oblige de les traiter séparément

Premièrement, nous commençons par les problèmes d'ordre linguistique : il s'agit d'abord de cette différence entre la structure de la phrase arabe surtout l'arabe classique et la phrase française, citant à titre d'exemple :

\section{(البيعان بالخيار ما لم يتفرقا) \\ (L'acheteur et le vendeur garde toujours le choix jusqu'à ce qu'ils se séparent et quittent la séance de vente) ${ }^{(1)}$}

Il se compose de 5 mots : un sujet (prédicat), un attribut et une restriction, Le Hadith emploie le terme (Bayaan) pour traduire (deux vendeurs) alors qu'il s'agit d'un seul vendeur et acheteur dans la langue arabe, Il y a ce que nous appelons (la dualité) par prévalence, On dit deux lunes au lieu de dire une lune et un soleil pour désigner un et une jolie. Donc

(1) (Hadith prophétique relative à la vente qui se trouve dans les livres de Fiqh, pris du livre Al-Mawardy : Al-Ahkam El-Sultaneya et traduit par Ostrorog . 142 ) 
on fait la distinction mais lorsqu'on dit deux lunes ou deux soleils cela veut dire qu'ils sont ensemble; c'est un procède linguistique arabe qui n'a pas d'équivalent dans la langue française, Pour résoudre le problème il faut comprendre la signification de l'énoncé en arabe et transmettre cette signification d'une façon compréhensible dans la langue française. Le mot (belkheyar) si on le traduit littéralement on va dire avec le choix ou par le choix alors que le vouloir dire de ce terme est diffèrent ; le contexte exige que les deux parties du contrat de vente restent libre à choisir et a décider tant que le négoce n'est pas accord conclu. C'est pourquoi on le traduit par ( gardent leurs choix ), La restriction pourrait être traduite en français par ( tant qu'ils ne ), Iil est Claire que la structure est différente, car en arabe il y a deux outils de négation alors qu'en français il y a une locution de condition, mais le vouloir dire est rempli dans le sens de ( se séparer ) pour désigner leur séparation et leur départ après la conclusion du négoce, c'est pourquoi l'écrivain la traduit par quitter La scène de la vente .

( il y a des problèmes propre a la signification juridique, les difficultés de l'analyse du sens sont certaines, qu'ils s'agissent de ses aspects logiques ou ses aspects culturels $)^{(1)}$

De ce qui précède, nous pouvons dire qu'il faut faire recours aux procédés linguistiques des deux langues, compris et fréquents chez les lecteurs pour surmonter les problèmes d'ordre linguistiques.

Deuxièmement , Passons maintenant aux problèmes d'ordre socioculturel, Il s'agit de ces termes indissociables du milieu culturel et social, on dit en arabe par exemple : ( mohtasseb ), ( sayrafy ), ( waly ), ( cadi ), ( cadda ), ( aamal ), et d'autres termes cités dans le livre d'AlMawardy : Al Ahkam Al-Sultaneya et interprété par Al-Assanhoury dans son œuvre (Le califat).

Si nous traduisons ces termes en français nous pourrons tomber dans le piège de l'incompatibilité, D'abord le mot (Mohtasseb) pour bien comprendre son sens, il faut bien connaitre sa fonction, Il s'agit d'une personne nommée par le gouverneur, pour surveiller le marché, détecter les bonnes et mauvaises marchandises et découvrir les fraudes. Pour le traduire ; l'écrivain a eu recours aux termes (contrôleur du marché ou bien (superviseur du commerce. Pour le mot (Al-Sayrafy), il s'agit de celui qui vend la monnaie et faire les échanges, Si nous le traduisons par le banquier ; la traduction ne sera pas exacte car le banquier a d'autres charges en plus.

(1) Brèves notes sur les problèmes de la traduction juridique - Revue internationale de droit comparé Année 1986 Volume 38 Numéro 2 pp. 349 
Donc on suggère le traduire par (le monnayeur). Le mot (wali) le wali c'est un agent, grand fonctionnaire, investi par le calife pour gérer les affaires d'un pays ou d'un département musulman, Son pouvoir n'est pas indépendant du gouvernement central. Donc le traduire par gouverneur est excessif, Le traduire par préfet est réducteur. Donc Al-Assanhoury a maintenu le terme (wali) ou nous pouvons employer le terme lieutenant mais il y a ici un risque de confusion entre le civil et le militaire. Le mot (Cadi) en arabe n'est pas tout à fait le juge en français ; Le cadi en islam est un savant jurisconsulte qui a le droit de juger d'après ses propres efforts de déduction. Alors que le juge en français ne fait qu'interpréter la loi, Le mot ( kadaa ) pourrait signifier soit justice soit département, Nous disons à l'époque ( kadaa el Bassra ), Le mot (aamal) dans le sens d'action ou œuvre n'est pas voulu ici chez Al-Mawardi mais il signifie dans le droit administrative ( le banlieue ) .

Par conséquent, nous pouvons dire que si nous ne comprenons pas le contexte socioculturel à l' origine de l'élaboration de quelques termes, nous pourrons avoir le risque de traduire certains termes d'une façon inexacte.

Troisièmement : en ce qui concerne les termes qui ont une spécificité religieuse et les termes relevant du Coran et de la Sunna et qui ont des significations quasiment spécifiques, nous trouvons par exemple et parmi des milliers d'autres selon le verbe (Gharar) et les mots (ekala), (soht), (tawleya), Ce sont des termes qui se trouvent dans la Sunna du prophète Muhammad (SBL) et interprètes dans le livre de ( Fekeh El-Sunna ) d'AlSayed Sabek .

Ce sont des termes de spécificités religieuses, en plus de leur caractère civil. Le verbe (Gharar) dans le sens de la tromperie et la ruse ne peut être compris que dans le cadre de la spécificité religieuse car, le prophète Muhammad (SBL) fait une certaine liaison entre tromper quelqu'un et trahir les ordres d'Allah. Preuve à l'appui ; le châtiment dans l'au-delà est plus dure que la peine dans l'ici-bas. Le mot (ekala) ne signifie pas (renvoi) ni (démission) mais il signifie qu'un acheteur accepte de rendre les marchandises au vendeur regrettant sans clause disciplinaire et cela se trouve dans l'interprétation d'un Hadith du prophète Muhammad (Salutations et Bénédictions soient accordés sur lui)

$$
\text { ( من اقال نادما بيعته اقاله الله عثرته يوم القيامة ) }
$$

Le mot (Soht) est cité plusieurs fois dans le Coran dans plusieurs versées, C'est un mot solitaire, Il n'y a pas de verbe ni d'adverbes ni rien qui équivale ce mot, il signifie la consommation des biens illicites . 
Celui qui n'a pas une culture religieuse n'en pourra pas comprendre le sens.

Le mot ( Tawleya ) dans le livre ( Le Fiqh de la Sunna) )- deuxième chapitre- d'( Al-Sayed Sabek ) a deux sens dans le droit musulman; selon le chapitre en matière de la vente, il signifie vendre sans gain, remise de la marchandise du même prix de l'achat et dans le chapitre du gouvernement signifie l'investiture.

En guise de conclusion; nous pouvons dire que nous avons besoin de traduire le droit musulman pour un bon nombre de raisons mais cette traduction n'est pas une mission facile. Nombreuses sont les difficultés de la traduction spécifique et pleins de pièges, Cela nous mène à une question très importante à savoir ; comment surmonter et faire face à ces problèmes?

Tout d'abord, le traducteur devra faire beaucoup des recherches, et avoir une formation solide et méthodique pour bien maitriser les particularités du langage juridique. Ensuite, il faudra consulter des œuvres relatifs à la culture sociale de l'époque et du people concernés, Aussi, pour résoudre les problèmes d'ordre linguistiques, il faut prendre en considération les différenciations de structure et de rhétorique entre les deux langues cible et source. En outre, afin d'éviter les problèmes d'ordre socioculturel ayant des dimensions religieuses, il devra mettre en considération que la sphère culturelle qui est à l'origine de l'élaboration des termes n'est pas la même dans les deux langues, nous pouvons ajouter également que la dimension religieuse est omniprésente dans le patrimoine islamique et presque absente ou sous-entendue dans le droit moderne, Enfin, il faut établir un glossaire des termes et des expressions spécifiques avant de procéder effectivement a les traduire .

Deuxième partie

(Application sur le (Califat) d' (Al-Sanhouri)

Premier chapitre

(Le droit administratif)

$\mathrm{Au}$ cours de la deuxième partie nous allons traiter une application sur le livre (Le Califat) de (Abdel Razak Al-Sanhouri) .

Abd el Razak Al-Sanhouri est le juriste le plus célèbre dans le monde arabe a son époque et probablement jusqu'à présent, Il a eu son doctorat d'Etat de l'université de Lyon sous la direction du doyen Eduard Lambert, Il a préparé deux thèses dont la principale est intitulée (les restrictions contractuelles a la liberté individuelle du travail dans la jurisprudence anglaise), étude purement juridique. La deuxième thèse est intitule (Le califat et son évolution vers une société des nations orientales. Il était 
professeur de droit à l'université du Caire et chef du Conseil d'e l'État jusqu'aux années 1960.

C'est lui qui a mis les constitutions, le droit pénale, le droit administratif, le droit commercial, d'un bon nombre des pays arabe dont le Koweït en 1968 juste avant sa mort

Passant maintenant à son œuvre (le Califat), Dans ce livre Al-Sanhouri traite la question du califat qui venait à l'époque de se déchoir en 1924, Il a subdivisé son œuvre en deux parties ; la première est théorique, la deuxième est historique. Dans la partie théorique AL-Sanhouri se trouvait dans l'obligation de faire référence aux sources du patrimoine islamiques: le Coran et la Sunna et surtout les livres u Fiqh islamique comme les statuts gouvernementaux d'Al-Mawardy, la Mawakef d'Al-Egy , Al-Makased d'AlGhazali et la prolégomènes d'ibn Khaldoun parmi d'autres .

Il se trouvait également devant le problème de la traduction et de la traduisibilite' des termes du droit musulman, Il a déployé des efforts immense et remporté le succès dans la plupart du temps ; cela est dû a sa connaissance très vaste en termes de sciences religieuses; sa maitrise de la langue Arabe et sa distinction dans la langue française, Ce sont bien les outils pour effectuer une traduction spécifique.

Dans la première partie de son livre (Le califat), Al- Sanhouri appréhende l'institution du califat tout de suite après la mort du prophète Muhammad (SBL), Il a cherché dans le Coran et la Sunna la source de base du califat mais il ne l'a pas trouvé. Il a estimé que cette institution est basée sur le consensus (el-ejmaa) ; la source de législation qu'il considéré la plus vaste, la plus souple et adaptable aux survenues de la société.

Il traite des questions à la fois juridiques, religieuses, historiques et administratives. Il se sert du lexique juridique pour transposer les termes du droits musulman et essaie dans la mesure de possible de remplir le vouloir dire des doctes musulmans.

Il s'est bien servi de certaines traduction et ouvrages arabes dont en tête ; Al-Ahkam Al-Sultaneya d'Al-Mawardy et traduit par le comte Léon Ostrorog sous le titre de (statuts gouvernementaux) ainsi que les ouvrage relatifs au califat publies en anglais, du fait qu'il maitrisait également la langue anglaise.

Dans le premier chapitre, nous entendons traiter les termes du droit administratif musulman selon le livre d'Al-Sanhouri malgré le fait que le droit musulman ne connait pas cette classification a ce temps la .

Le droit Administratif est le domaine qui traite tout ce qui est administration à partir du chef d'Etat jusqu'au petit fonctionnaire dans le 
droit musulman, Il existe des chapitres relatifs à l'administration comme le califat, l'investiture du calife, le fonctionnement du califat, le trésor publique, les impôts, les taxes, les frais douaniers, les fonctionnaires de l'état, le vizir les agents publiques, leurs investitures et leurs déchéances, le corps de la magistrature et le fonctionnement de la justice. Malgré cette littérature abondante il n'y a pas de code administratif dans le droit musulman. Les questions de ce domaine sont éparpillées dans les livres du Fiqh.

Par conséquent, la mission d'Alsanhouri visant à dégager les termes administratifs des tréfonds des livres du Fiqh n'étaient pas bien aisée, sa mission de les traduire étaient de la même difficulté.

Nous allons commencer par traiter le gouvernement dans le droit administratif,

Le gouvernement dans le droit musulman vient sous plusieurs termes, on dit par exemple : Al-Sultan, Al-Wilaya, Al-Molk, Al-Wali ...etc.

(Le Calife effectivement est le Wali d'Al-Molk il est aussi le wali ou le (wassi) des biens administratifs) ${ }^{(1)}$

Al-Sanhouri a utilisé le premier Wali dans le sens du calife mais le deuxième wali est dans le sens du gardien des biens.

Il est fréquent qu'Al-Sanhoury traduise ces termes comme suit dans son livre : il emploie le gouvernement pour désigner l'origine (Nezam El Hokm), il a gardé le terme (Wilaya) et il n' a pas employé le terme (pouvoir) parce que (la wilaya) est plus vaste que ( le pouvoir) car, dans le droit moderne tout pouvoir a des limites infranchissables alors que la wilaya du calife est illimitée exception faiet pour les quatre premiers califes suite à la mort du prophète

( Le califat despote d'un pouvoir illimité et absolu, en fait avec la transformation du califat régulier en une monarchie héréditaire et tyrannique, il a assumé des pouvoirs que le droit ne lui a point accordé ; c'est à la suite d'une évolution historique fatale comparable à celle qui s'est produite dans l'histoire de Rome, quand elle s'est transformée d'une république en une empire, L'état arriéré des institutions sociales et politiques ont mis fin rapidement à la courte période de l'admirable démocratie des quatre premiers califes )

L'empire musulman compose des éléments hétérogènes et disparates, était destinée à tomber sous la puissance et 'absolutisme du premier

(1) Al-Sanhouri, Le Califat, p.166 ; l'administration (la gestion) 
homme énergique et sans scrupules, qui par la force ou par la ruse, saurait s'emparer du pouvoir et fonder une dynastie. $)^{(1)}$

Il emploie pour le terme (tawleya) l'équivalent (investiture) qui exige un pouvoir plus puissant que celui de l'investi, parce qu'il n'existe pas le terme pertinent, il a eu recours à un terme rapprochant.

Al-Sanhouri parle de l'idéal adopté et appliqué dans le droit moderne à savoir ; c'est la communauté qui doit investir le roi, chose qui n'est pas la règle dans l'histoire de l'islam. C'est pourquoi Al-Sanhouri parle de ce qu'on appelle actuellement (la nomination) et à l'époque (welayet el ahd) (le père nomme son fils calife après sa mort ) et aussi ( le gouverneur par force) par traduire le terme ( Al-hakem el motaghaleb). Actuellement il existe un terme juridique très fréquent dans la presse pour désigner le gouverneur qui vient au pouvoir par un coup d'état (l'homme fort), Nous croyons que ce terme est plus adéquat que le gouverneur par force.

(Le calife est chargé d'assurer la justice, la sécurité, la défense des frontières, la gestion financière, la nomination des fonctionnaires, et enfin l'administration) $)^{(2)}$

Parmi les termes utilisés il y a aussi le terme (vizir); un terme qui a connu son crépuscule officiellement après l'époque des quatre premiers califes et devenu un poste prestigieux, Al-Sanhouri préfère maintenir le terme oriental francisé (vizir) car, le vizir oriental n'est pas tout à fait (le ministre) dans le droit moderne. Il n'appartient pas à un cabinet, ni à un parti politique, il s'agit plutôt d'un seul vizir qui dispose d'un pouvoir étendu et pareil à celui du Calife sauf dans trois cas exceptionnels.

(Le poste de ministre n'a pas été reconnu officiellement pendant le califat régulier des quatre premiers califes, bien qu'il ait existait en fait. C'est avec la dynastie que le titre de (vizir) apparait, le titre prend une importance considérable avec les abbasides. En général, tout ce que le calife, peut valablement faire le vizir peut le faire valablement, si ce n'est trois choses : la première est de désigner un successeur, le deuxième est de résigner ses fonctions, le troisième est de révoquer un fonctionnaire nommé par le calife) ${ }^{(3)}$

Pour le terme (omal) qui désigne en arabe un corps de haut fonctionnaire qui comprend le gouverneur de province, les responsables du

(1) Ibid., p. 172

(2) Ibid., p. 155

(3) Al Sanhouri, Op. Cit. p. 161 
trésor publiques et les chefs de l'armée, Al-Sanhoury préfère le terme (agents des califats)

(Les agents du calife sont ou des agents religieux comme les imams de la prière, les walis de pèlerinage, les agents chargés de la perception de l'aumône ou des agents politiques, comme les chefs de la police, les généraux de l'armée, les juges et les magistrats ) ${ }^{(1)}$

Nous croyons que ces termes traduits par Al-Sanhouri remplissent bien le vouloir dire des doctes musulmans.

Deuxièmement : nous passons à la fiscalité dans l'islam ; un dossier très chargé que les doctes musulmans ont en beaucoup à dire.

Nous allons aborder des termes économiques dans le droit musulman dont Al-Sanhouri a fait une traduction adéquate dans le but de transmettre le vouloir dire des juristes de l'islam.

Notre écrivain a employé le terme (kaharaj) c'est à dire cet impôt a prélever sur la récolte des terrains cultivables, Il emploie le terme (dixième) pour désigner l'impôt (oshr) qui est prélevé a certains terrains autre que ceux de (kaharaj), Il emploie le terme (trésor publique) pour designer (bat el mal) ou (la maison des biens).

(Le mot (zakat) est un système obligatoire : aide financière impose' au riche au profit des pauvres dans le but de purifier religieusement leurs biens, le mot Zakat signifie littéralement la purification dans un verset coranique on lit (prenez une aumône obligatoire de leurs biens pour les purifier) $\left.{ }^{(2)}\right)$

(Le mot (Djezyah) a le même sens que (la zakat) mais pour les non musulmans, c'est un devoir catégorique individuel. Les musulmans et les non-musulmans qui ne remplissent pas ce devoir de charité doivent être forcés a le faire, s'ils résistent, le califat peut aller jusqu'à les combattre suivant le précèdent d'Abou Bakr dans ses guerres contre les apostats $^{(3)}$.

Le terme (aumône) qui traduit (la zakat) est bien employé à condition de y ajouter l'adjectif «obligatoire » ou "prescrite», Ainsi que l'écrivain a préféré de garder le terme (djizyah) qui n'a pas d'équivalent en français qui pourrait le traduire,

\footnotetext{
${ }^{(1)}$ Ibid., p. 157

(2) (Al-Sanhouri, Le Califat, p.151, l'aumône)

(3) Al-Sanhouri, Le Califat, p.151, citation tirée d'Al-Ahkam Al-Sultaneya d'Al-Mawardy, p. 99
} 
De même pour les termes (Al-Ghanaeem ), ( Al- fay') et ( $\mathrm{Al}-\mathrm{Awkaf}$ Al-khayreya ) qui n'ont pas d'équivalents également

( ̀̀ cote de la zakat il y a aussi (al-fay') ; les biens pris sur les ennemis sans livrer la bataille, et (Al-Ghanaeem) ; biens de l'ennemi pris après avoir livré une bataille, le cinquième de Al-Ghanaeem sont reparti aussi bien que (Al-Fay'), al awkaf al-khayreya (wakfs religieux) sont des fondations charitables dans un but religieux $)^{(I)}$

Il ne faut pas oublier qu'Al-Sanhouri a déployé des efforts grandioses pour traduire ces termes spécifiques dans le but de transmettre le véritable message de la religion.

Troisièmement : nous passons maintenant à une question très épineuse dont la traduction des termes pourrait être contre les notions, Le principe du (djihad) qui est toujours incompréhensible aussi bien pour les non musulmans que pour une grande partie des musulmans.

Selon Al-Sanhouri, le terme (djihad) désigne (effort) ou (lutte contre). Il pourrait désigner; déployer des efforts pour faire quelque chose ou lutter contre le Diable, les mauvais penchants de l'âme, lutter contre les hérétiques ou bien les ennemis. Donc, le dJihad n'est pas la guerre sainte comme on a l'habitude de le traduire, car, c'est une traduction qui ne remplit jamais le vouloir dire d'une part et reflète effectivement le contraire voulu par les ennemis de l'islam qui ont des intérêts à falsifier l'image de la religion d'autre part.

La guerre sainte est un terme occidental qui remonte à l'époque des croisades qui prétendaient mener la guerre sainte dans le but de récupérer le Saint Sépulcre, Alors, le caractère sacrée de la guerre n'est pas un produit islamique.

(Le Califat doit donc pour remplir son devoir concernant son devoir pour remplir la djihad; organiser des missions religieuses aux pays nonmusulmans et dissiper les erreurs grossières qu'on répand malheureusement, quelquefois de mauvaise foi contre l'islam, au moyen d'une propagande énergique.

Dans un seul cas, celui d'une agression armée contre les pays musulmans, il doit prescrire la guerre défensive a tous les musulmans capable de combattre, ceux-ci sont tenus, par un devoir catégorique individuel, de répondre à l'appel et de défendre leur patrie) ${ }^{(2)}$

Selon le califat, le djihad en islam a pour finalité sublime ce qui suit :

\footnotetext{
(1) Al Sanhouri, Op. Cit, p. 151

(2) Ibid., p. 150
} 
1-Defendre les territoires musulmans contre l'agression des autres, ce qui est un droit assuré à tout le monde.

2-Assurer et garantir la liberté de conscience

3-Defendre le monde entier contre l'agression en ce qui concerne ceux qui ont été expulsés de leurs demeures. Comme le Coran l'affirme :

(Si Allah ne repoussait pas les gens les uns par les autres, les ermitages seraient démolis ; ainsi que les églises, les synagogues et les moqués ou le nom d'Allah est beaucoup invoque, Allah soutient, certes, ceux qui soutiennent sa religion) -Allah est assurément fort et puissant.) ${ }^{(l)}$

Alors, le djihad islamique est au profit de tout le monde car, il s'agit d'une guerre noble qui défend les droits de l'homme.

Al-Sanhouri préfère au lieu du terme (djihad) celui de combat ou mobilisation tout en excluant celui de la guerre sainte parce que ce terme a une connotation péjorative car, il signifie que les musulmans tuent au nom d'Allah, chose qui n'est pas correcte .

C'est l'Occident qui a inventé le terme et c'est bien lui qui en a condamné l'islam.

( si avec le progrès de la civilisation, on trouve d'autres moyens plus efficaces pour répandre la religion, on doit certainement y recourir, à l'exclusion de la guerre, or dans notre temps, une religion se répand beaucoup plus efficacement par la persuasion et la conviction par la propagande des mission religieuses et même commerciales, on en trouve un exemple frappant dans la propagation de l'islam en Afrique et en Asie $)^{(2)}$

(Déjà du temps d'Ibn Khadoun la Djihad dans sa forme primitive $n^{\prime e ́ t a i t ~ p a s ~ q u ' u n ~ s o u v e n i r ~ h i s t o r i q u e) ~}{ }^{(3)}$

Avec le temps et l'évolution de la raison et de l'esprit, les califes ont trouvé qu'ils n'ont pas besoin du djihad pour défendre l'islam ni pour la prolifération de la religion ; l'islam est déjà fort et il existe d'autres moyens pour répandre la religion dans les quatre coins du monde ttelles les missions religieuses et transactions commerciales

${ }^{(1)}$ La Sourate (Al-Hajj) (verset 43) :

(2) Al-Sanhouri, Le Califat, p.149, la guerre sainte

(3) Al- Sanhouri, Le Califat, p.150, la guerre sainte, citation tirée des prolégomènes d'Ibn Khaldoun page 251 


\section{Deuxieme Chapitre}

\section{(Le droit Judiciaire)}

Le droit judiciaire dans le sens moderne est le code qui organise le pouvoir judiciaire, Malgré le fait que l'idée de la séparation du pouvoir trouva son apparition pour la première fois au 18eme siècle dans « l'esprit des lois » de Charles Montesquieu qui était lui-même un magistrat,, cette idée a été connu dans l'islam tout au long de son histoire Mais il prenait une autre forme, le calife retenait les deux pouvoirs exécutif et législatif alors que le pouvoir judiciaire était toujours indépendant comme l'affirme AlSanhouri .

(La séparation des pouvoirs dans l'islam est donc, toute faite dès le début, il n'y a pas besoin d'attendre un réformateur pour l'accomplir) ${ }^{(1)}$

Al-Sanhouri affirme également que le magistrat était conscient d'avoir prendre son pouvoir non pas du gouverneur mais de l'établissement de la justice et de l'agrément populaire.

Il est vrai que la nomination des juges était la mission du calife mais il ne s'intervenait pas dans leur travail.

(Le Calife, le vizir et le gouverneur général peuvent nommer les juges et les révoquer, il ne se trouve pas révoqué du fait que la magistrature du calife prend fin) ${ }^{(2)}$

(Les califes ont pris l'habitude de respecter l'indépendance des juges ; c'est ainsi qu'on raconte qu'Omar le deuxième Calife a refusée d'annuler une sentence judiciaire rendue par le juge Abou-Al-Dardaa, bien que cette sentence ait été contraire a son propre avis) ${ }^{(3)}$

Le Cadi en islam n'est pas tout à fait l'équivalent du juge dans le sens actuel, le Cadi en islam doit appartenir à une école juridique, il est hanafite, shaféite, malékite ou hanbalite alors il était un savant, un jurisconsulte et un mufti.

(Le juge a des fonctions judiciaires proprement dites et des fonctions judiciaires quasi-judicaire, il englobe la justice civile, et la justice pénale. Il assure l'exécution des sentences judiciaires, il nomme les délègues, il prend des mesures pour s'assurer que les témoins remplissent les conditions légales, il observe en rendant la justice l'impartialité parfait, le juge exerce aussi une certaine compétence en ce qui concerne les

(1) Al-Sanhouri, Le Califat, p.142

(2) Al-Sanhouri, Le Califat, p.170, le pouvoir judiciaire, nomination et révocation du juge

(3) Al-Sanhouri, Le Califat, p.168, le pouvoir judiciaire, citation tirée du prolégomènes d'Ibn Khadoun, p, 245 
incapables, le mariage, les biens constitués en Waqfs, l'exécution des testaments et l'interdiction des prodigues) ${ }^{(1)}$

Le juge actuel ne fait qu'appliquer les lois et dans quelque pays interpréter les règles juridiques, les lois et les principes.

En Islam, la conception d'audience qui se compose de trois magistrats n'existe pas mais il avait un seul juge qui arbitre les litiges.

Le parquet public (général) chargé des investigations et enquêtes n'existe pas en Islam, c'est le juge qui fait toute cette structure magistrale très compliquée qui n'était pas exigible à l'époque. La hiérarchie juridictionnelle n'avait pas lieu en Islam : un seul jugement une fois prononcée est définitif, c'est parce que le Cadi examinait soigneusement les faits et les questions et ne prononçait le verdict qu'après une certitude sans équivoque.

(Les musulmans connaissent la police sous le nom de (choral), elle est limitée à la mise en accusation des pénalités; compétence analogue à celle du ministère public de nos jours) ${ }^{(2)}$

La police ne jouait aucun rôle en matière des investigations, sa mission se restreignait à l'arrestation et l'exécution des jugements. .Les conditions des détenus étaient aussi parmi les fonctions et le travail du Cadi.

De même, une fonction était très important aussi, c'était celle du Cadi des Cadis ou bien ( le juge suprême ), il s'agit du premier Cadi de l'état équivalent en quelque sorte du ministre de la justice responsable des cadis départementaux .

Nous allons effectuer maintenant une comparaison entre le pouvoir judiciaire en islam et celui en droit moderne :

Tout d'abord, en Islam l'extraction des sentences et jugements passe par un processus polyvalent et sensible. Il existe des grandes lignes qui doivent être compatibles complètement aux finalités de la Charia et qui constituent la philosophie même de la législation. Il s'agit de cinq principes fondamentaux qui sont à savoir : la préservation de l'âme, la religion, la raison, la filiation ou l'honneur et les biens.

(L'exercice d'un droit ne peut se produire que dans le but pour lequel ce droit a été accordé comme le hadith prophétique : aucune obéissance n'est due à l'homme qui désobéit a son Créateur) ${ }^{(3)}$

(1) Al Sanhouri, Le Califat, p.170, 171 - le pouvoir judiciaire

(2) Al-Sanhouri, Op.cit. , p.156, le pouvoir exécutif

(3) Al-Sanhouri, Le califat, p, 175, l'abus du pouvoir 
(La Charia ou Le droit Musulman est construit sur des finalités et sur les intérêts des gens dans leur vie actuelle et future. il est de toute justice et de toute pitié, de tout intérêt, et de toute finalité. Par conséquent, toute solution qui sort de la justice a l'injustice, de la pitié a son contraire, de l'utile au nuisible, de la finalité a ce qui n'a pas de but, ne sera plus de la Charia, même si elle rentre dans la lettre du Charria) ${ }^{(1)}$

Ces principes clés sont déduits des sources de la législation; au premier abord il y a le Coran qui nous donne les grandes lignes et les prescriptions majeurs en plus certaines sentences détaillés, Vient au deuxième chef ; la Sunna ou bien les traditions prophétiques. Nous pouvons dire que la Sunna a un double rôle : d'une part, elle détaille les prescriptions majeurs et les interprète, et d'autre part, elle institue d'autres sentences.

La troisième source de législation est le consensus (ejmaa) ; il s'élabore à travers une assemblée des doctes qui examine une affaire pour aboutir à une solution communale.

Al-Sanhouri considère que cette assemblée représente la plus grande autorité qui reflète la souveraineté de la communauté.

La quatrième source est l'analogie, c'est-à-dire : il y a un fait survenu dépourvu d'une sentence légale, comment le juger?

Il faut adjoindre d'un autre fait ayant déjà une sentence en raison de la causalité commune, citant à titre d'exemple ; le vin est prohibé car, il camoufle la raison, Les drogues produisent le même effet. Alors, ils sont prohibées et interdits par analogie au vin, et il y a d'autres sources subsidiaire comme l'option préférentiel, la quête de l'intérêt .... Etc.

Dans l'application de ses principes, les écoles juridiques musulmanes parfois se convergent et parfois se divergent.

Le Cadi, docte lui-même adopte une école juridique, il a le droit de juger en fonction des dires des doctes de son école, de l'un deux ou bien en vertu des opinions des autres écoles, Alors, nous pouvons dire que c'est lui qui choisit la sentence qu'il la juge adéquate, c'est lui qui l'interprète et l'applique .

Passant maintenant à ce qui concerne le droit moderne, il y a trois pouvoirs : le pouvoir exécutif dont en tête le chef de l'Etat, le pouvoir législatif représenté par le parlement qui décrète les lois desquelles s'engage le pouvoir judiciaire d'une façon impérative.

(1) Al-Sanhouri, Le Califat, p.177 - abus du pouvoir 
A l'inverse, du Cadi islamique, l'action du juge actuel est limitée dans le cadre des lois qu'il ne fait que les appliquer, et lorsqu'il tombe dans une erreur interprétative il y a d'autres juridictions qui pourraient soit ratifier ses jugements, soit les corriger.

En ce qui concerne les sources de la législation du droit positif, nous trouvons que les textes sacrés sont absents.

Ils adoptent premièrement ce que Rousseau appelle (La loi naturelle) ; c'est à dire la loi tirée de la réalité et ils prennent en considération aussi le droit roman surtout pour les règles de base et ils promulguent des lois en fonction des exigences de la réalité vécue.

Ils ne prennent pas toujours en considération les finalités universelle ; chaque pays décrète les lois qui conviennent aux exigences locales, il y a une distinction remarquable entre les divers droits : droit pénal, droit administratif, droit commercial, droit de procédure, droit civil, droit constitutionnel, droit de procédure ....etc.

Malgré la présence de beaucoup de ressemblances entre plusieurs lois occidentales et leurs homologues islamiques, l'écart reste très large du point de vue de la philosophie des deux camps.

De ce qui précède apparait le rôle du traducteur dans l'interprétation des termes difficiles et spécifique du droit musulman.

Al-Sanhouri a bien réussi à transposer le terme arabe et son équivalent en français, Nous avons donné des exemples au cours de notre développement de ce chapitre comme : La Sunna, l'Ejmaa, Al Keyas , Mogtahed, Mazhab, Makased el chariaa, Hefz el nafs .

Sa mission est bien accomplie où il a pu transmettre le vouloir dire des doctes musulmans, chose qu'il a bien éclaircie en termes du message de sa mission.

Cette institution est indissociable de l'esprit même de l'islam, tous les musulmans sont solidaires en termes de la défense des droits collectifs et de préserver l'intérêt commun.

((Al-Hisbah est une justice sommaire dans des questions d'une importance secondaire, notamment dans celles des poids et des mesures) ${ }^{(1)}$

Le Mohtasseb (le contrôleur) est une personne nommé par le Calife ou le gouvernement d'une province pour inspecter et superviser le marché.

Il joue le même rôle de la police dans le temps moderne. Cette personne travaille sous le contrôle du pouvoir judiciaire, Il y a une autre

(1) Al-Sanhouri, Le Califat, p. 171, limites de la wilaya du calife 
forme de Hesba qui pourrait être traduite par l'action communale : une personne qui tombe sur une erreur ou un délit qui menace la société et lui constitue un danger.

Bénévolement, et en attendant la rétribution auprès d'Allah, il passe une action devant le juge, ce dernier prononce un jugement, après les enquêtes nécessaires et oblige l'Etat à corriger la faute, arrêter le coupable et contourner le danger. Ce contrôle social s'inscrit dans le cadre d'une communauté dont les individus sont consolidaires.

Cette institution n'existe pas dans le droit moderne. À l'inverse dans le droit positif, pour intenter une action, il faut, soit avoir une qualité (une partie d'un contrat) ou ayant intérêt (celui qui subit directement en personne un préjudice à cause du délit.) Cela met en évidence la grande différence entre le pouvoir en islam et son équivalent dans le droit moderne.

Encore une fois, Al-Sanhouri nous émerveille par exceller la traduction des termes du droit musulman en français.

\section{Conclusion}

$\mathrm{Au}$ terme de cette recherche, nous pouvons affirmer que la traduction générale est un processus difficile, pénible, complexe et polyvalente.

La difficulté vient de la différence structurelle entre la langue cible et la langue source.

La peine vient du fait qu'il fait déployer tant d'effort pour essayer de transmettre le vouloir dire de l'écrivain.

La complexité est naturelle et normale car, la traduction exige une connaissance bien approfondit en plusieurs disciplines linguistique et paralinguistique.

La connaissance de la grammaire en est indispensable, la morphologie qui s'occupe de la dérivation est d'une importance capitale.

La rhétorique et les figures de styles doivent être maitrisées par le traducteur pour qu'il puisse transmettre bien le message avec toutes les subtilités stylistiques.

Quant à la traduction spécifique, corpus de notre recherche, surtout la traduction juridique, elle exige en plus les connaissances et les compétences susmentionnés, le perfectionnement du contexte juridique et une érudition exemplaire dans la discipline, car, les spécialistes sont les plus connaisseurs des notions et conceptions des termes spécifiques et sont les plus capables d'en remplir le sens.

Notre corpus est le livre du Califat de Abdel-Razak El-Sanhouri. Il s'agit d'une thèse de doctorat d'état publiée en français qui porte sur un thème purement islamique et orientale. 
Le thème du Califat évoque plusieurs sujets à traiter surtout dans le domaine juridique.

Etant donné que la jurisprudence islamique ne connaissait pas cette classification juridique moderne (civil, pénale ......etc.) et que les rameaux juridiques pourraient en être déduits par déduction de cet amas juridique enchevêtré, la tache de Sanhouri visant à déceler les différents genres de droit n'était pas facile. Il a essayé de montrer que les livres de Fiqh musulmans sont abondants des sentences et lois qui équivalent pour ne pas dire prévalent sur les lois modernes.

Notre travail dans cette recherche était de bien examiner l'itinéraire du Sanhouri dans son exposition et comment il a réussi à traduire et corriger la traduction des plusieurs passages du patrimoine musulman.

Il a été précurseur en termes de la traduction de plusieurs idiomes purement patrimoniaux islamiques à tel point qu'il se fit un pont qui relie deux cultures différentes : orientales et occidentales.

Avant de terminer notre conclusion nous pouvons dresser une liste de recommandations pour une bonne traduction :

1-La maitrise de la langue cible

2-Être livré et versé dans la discipline

3-L'equivalence conceptuel entre les termes des droits musulmans et ceux des droits positifs n'est pas toujours fourni

3-Trop souvent, il faut faire recours à une traduction rapprochante pour représenter le vouloir dire des doctes musulmans.

$\mathrm{A}^{\prime}$ la fin de notre recherche nous posons une question importante :

Pourrait-on avoir un dictionnaire juridique double - français /Arabe Arabe/ Français qui met en parallèle le terme juridique et son équivalent dans le droit musulman, tout en démontrant la différence des points de vue de la notion? Quels sont les procédés d'ordre lexicologique et traductologique requises pour effectuer un tel travail colossal ? (Un guide juridique islamique orienteur)

\section{Bibliographie}

\section{Corpus}

1- Assanhoury (A.), Le califat, son évolution vers une Société des nations orientales, in- $8^{\circ}$, xvi-627 pages. Paris, Geuthner, 1926

\section{Références sur la traduction juridique}

Manual de la conjugación del español, Octavio Santana Sarez, Francisco Carreras

2- Ruidavets, Zenon J. Hernandez Figueroa, José R. Pérez Aguiar, Gustavo Rodríguez Rodríguez. \ 
3- Manual de la interpretación y taducción, Mario león $>$ Traducción y Traductología, Introducción a la traducción, Ampara Hurtado Albir

4- $>$ www.erudit.org $>$

5- Dictionnaire LAROUSSE $>$ Diccionario de la Real Academia Española (RAE) $>$

6- Dictionnaire bilingüe Larousse $>$ Literalidad y dinamicidad en el discurso económico, M. Teresa Cabré, Carme Bach,

\section{Références bibliographiques}

7- BALlARD, M. (1995): De Cicéron à Benjamin. Traducteurs, traductions, réflexions, Lille, Presses universitaires de Lille.

8- BEAUPRÉ, M.(1987):"La traduction juridique. Introduction". 28 Cahiers de droit. Québec BEAUPRÉ, M. (1986): Interpreting Bilingual Legislation. Toronto, Carswell.

9- BOWERS, F. (1989): Linguistic Aspects of Legislative Expression. Vancouver. CERTOMÀ, G.L. (1986): "Problems of Juridical Translations in Legal Science". Law and Australian Legal Thinking in the 1980s. Sydney. COORAY, L.J.M. (1985):

10- Changing the Language of the Law. The Sri Lanka Experience. Québec, Presses de l'Université Laval. CORNU, G. (1990): Linguistique juridique. Paris.

11-CRÉPEAU, P. (1993): "L'affaire Daigle et la Cour suprême du Canada ou la méconnaissance de la tradition civiliste". Mélanges Germain Brière. E. Caparros (éd.).

12- Montréal, Wilson \& Lafleur. DANET, Brenda (1980): "Language in the Legal Process". Law and Society Review 14: pp. 445-564.

13-DAVID, R. et JAUFFRET-SPINOSI C. (1992): Les grands systèmes de droit contemporains, $10 \mathrm{e}$ éd., Paris. DELISLE, J. et WOODSWORTH, J. (dir.): Les traducteurs dans l'histoire, Les Presses de l'Université d'Ottawa et Editions UNESCO, 1995.

14-DIDIER, E. (1990): Langues et langages du droit, Montréal, Wilson \& Lafleur. FOCSANEANU, L. (1971): "Les langues comme moyen d'expression du droit international". Annuaire français de droit international. 16, Paris.

15- FRANÇOIS, F. (1968): "Le langage et ses fonctions". Le Langage, vol. 25 l'Encyclopédie de la Pléiade, Paris. GÉMAR, J.-C. (1995): Traduire ou l'art d'interpréter, t. II, Langue, droit et société: éléments de jurilinguistique. Québec, Presses de l'Université du Québec. 
16-GÉMAR, J.-C. (1990): "La traduction juridique: art ou technique d'interprétation?", Ottawa, Revue Générale de Droit (1987) 18 RGD, pp. 495-514. GÉMAR, J.-C. (1990): "Le langage du droit au risque de la traduction. De l'universel et du particulier" in Français juridique et science du droit, Bruxelles, Bruylant, 1995, pp. 123-154 (Textes présentés sous la direction de G. Snow et J.Vanderlinden).

17-GROFFIER, E. et REED, D. (1990): La lexicographie juridique. Québec. GROOT, G.R. DE (1987): "Problems of Legal Translation from the Point of View of a Comparative Lawyer". 28 Cahiers de droit. Québec.

18-HAGËGE, C. (1985): L'homme de paroles. Paris. HERBOTS, J.B. (1987): "La traduction juridique. Un point de vue belge". 28 Cahiers de droit. Québec.

19-KELSEN, H. (1962): Théorie pure du droit. Paris, Dalloz. KOVACS, Alexandre (1982): "La réalisation de la version française des lois du Canada", in Langage du droit et traduction.

20- J.-C. Gémar (éd.). Québec, Conseil de la langue française, pp. 83100. LANE, A. (1982): "Legal and Administrative Terminology and Translation Problems". Langage du droit et traduction.

21- J.-C. Gémar (éd.). Québec, Conseil de la langue française, pp. 219231. LERAT, P. (1995): Les langues spécialisées. Paris, Presses universitaires de France.

22- MAXWELL, P.B. (1976): The Interpretation of Statutes. 12th ed. by P. St. J. Langan, Bombay, Tripathi.

23- NADELMANN, K. H. et MEHREN, A. T. VAN (1966-1967): "Equivalences in Treaties in the Conflicts Field". 15 The American Journal of Comparative Law.

24-NEWMARK, P.P. (1982): "The Translation of Authoritative Statements: A Discussion". Langage du droit et traduction. Québec, pp. 283-299. NIDA, E. A. (1964): Toward a Science of Translating. Leiden.

25-NIDA, E. et TABER, C. (1971): La traduction: théorie et méthode. Londres.

26- PIGEON, L.-P. (1982): "La traduction juridique. L'équivalence fonctionnelle". Langage du droit et traduction. J.-C. Gémar (éd.). Québec, Conseil de la langue française, pp. 271-281. REY,A. (1991): Le Petit Robert. Paris.

27-ROULAND, N. (1991): Anthropologie juridique. Paris. SACCO, R. (1987): "La traduction juridique. Un point de vue italien". 28 Cahiers 
de droit. Québec. SARCEVIC, S. (1985): "Translation of CultureBound Terms in Laws". Multilingua, vol. 4, no 3, pp. 127-133.

28- SAUSSURE, F. DE (1916): Cours de linguistique générale. Paris (Ed. critie Tullio de Mauro. 1982).

29- SCHROTH, P.W. (1986): "Legal Translation". American Journal of Comparative Law. 34, suppl., pp. 47-65.

30- SOURIOUX, J.-L. et LERAt, P. (1975): Le langage du droit. Paris.

31-STEINER, G. (1992): After Babel. 2e éd., Oxford, Oxford University Press. "Traduction juridique (La)" (1987). Les Cahiers de droit. Québec, 28, 